\title{
Respiration rates of individual bovine in vitro-produced embryos measured with a novel, non-invasive and highly sensitive microsensor system
}

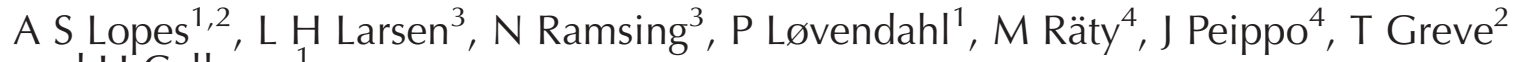 \\ and $\mathrm{H}^{\text {Callesen }}{ }^{1}$ \\ ${ }^{1}$ Department of Genetics and Biotechnology, Danish Institute of Agricultural Sciences, DK-8830 Tjele, Denmark, \\ ${ }^{2}$ Department of Large Animal Sciences, The Royal Veterinary and Agricultural University, DK-1870 Frederiksberg \\ C, Denmark, ${ }^{3}$ FertiliTech ApS, DK-8000 Aarhus C, Denmark and ${ }^{4}$ Animal Production Research, MTT- \\ Agrifood Research Finland, FIN-31600 Jokioinen, Finland
}

Correspondence should be addressed to A S Lopes, The Royal Veterinary and Agricultural University, Department of Large Animal Sciences, Section for Reproduction and Obstetrics, Dyrlægevej 68, 1870 Frederiksberg C, Denmark;

Email: AnaSJ.Lopes@agrsci.dk

\begin{abstract}
Oxygen consumption is a useful parameter for evaluating embryo quality, since it provides a valuable indication of overall metabolic activity. Over the years, several approaches have been used to measure the respiration rates of individual embryos, but a convincing method has not yet been reported. In this study, we introduce and have validated a novel high resolution microsensor technology to determine the respiration rates of individual embryos at different developmental stages. We have employed this technology to investigate the correlation between respiration rate and embryo morphology, diameter and sex. Following morphological evaluation, individual respiration rates of day $3(n=18)$ and day $7(n=60)$ bovine in vitro-produced embryos were determined. Of the measured embryos, 64 were lysed for sex diagnosis by PCR. Average respiration rates of day 7 embryos $(1.30 \pm 0.064 \mathrm{nl} / \mathrm{h})$ were 3.4 -fold higher than day $3 \mathrm{embryos}(0.38 \pm 0.011 \mathrm{nl} / \mathrm{h})$. On day 7 , the average respiration rate of quality 1 blastocysts was significantly higher than the respiration rates of the lower qualities. For both day 3 and day 7 embryos, respiration rates were directly influenced by embryo diameter but did not differ between sexes. These results have demonstrated that the novel microsensor technology can be used to accurately and rapidly (8 min) measure the respiration rates of individual embryos at different developmental stages. Respiration rates were only in partial agreement with embryo morphology, suggesting a slight discrepancy between these two methods in assessing embryo quality. It is likely that a combined assessment of embryo respiration and morphology would improve embryo classification and subsequent selection.
\end{abstract}

Reproduction (2005) 130 669-679

\section{Introduction}

Morphological evaluation is the main and usually the only technique used routinely to assess bovine embryo quality and subsequent viability, both in experimental work and in practice (Overström 1992, 1996, Boiso et al. 2002). However, such evaluation is subjective in itself, and consent is often not reached even among specialized and experienced personnel (Farin et al. 1995). Furthermore, selection of embryos based on morphology has been shown to be difficult, especially for embryos of intermediate morphological qualities (Farin et al. 1995, Hasler 1998). More objective selection criteria are therefore needed (Neuber et al. 2003, Scott 2003), and a better understanding of embryonic metabolism could be very useful in the development of new strategies for judging the quality and the developmental potential of individual embryos (Overström 1992, 1996, Overström et al. 1992).

Embryo metabolism has previously been assessed by measurement of nutrient consumption, such as glucose, pyruvate and amino acids (Leese \& Barton 1984, Leese 1991, Rieger 1992, Rieger et al. 1992, Rieger \& Loskutoff 1994, Gardner 1999, Gopichandran \& Leese 2003). However, oxygen consumption has been considered to be the parameter that provides the best indication of overall metabolic activity of a single embryo (Leese 2003), as adenosine triphosphate (ATP) production is mostly gener- 
ated by oxidative phosphorylation, a process in which oxygen has a key role (Thompson et al. 1996, Thompson 2000, Trimarchi et al. 2000a, Houghton et al. 2003, Leese 2003). Furthermore, earlier studies provided evidence that respiration rates of individual embryos directly correlated with quality (Abe \& Hoshi 2003) and with survival following embryo transfer (Overström 1992). Hence, quantification of oxygen consumption by single embryos, combined with a morphological assessment, could likely improve the criteria for selecting embryos and predicting their developmental competence.

Several approaches have been used to measure the respiration rates of preimplantation embryos. The first studies (Fridhandler et al. 1957, Mills \& Brinster 1967) used a Cartesian diver technique to measure the oxygen consumption of large groups of rabbit ova and preimplantation mouse embryos respectively. Subsequent studies on single human oocytes and blastocysts (Magnusson et al. 1986) and mouse blastocysts (Nilsson et al. 1982) employed spectrophotometric methods to indirectly measure the oxygen consumption of the embryo by determining the amount of oxyhaemoglobin converted to haemoglobin as oxygen was consumed. Overström (1987, 1992) developed a multi-channel apparatus with solidstate oxygen electrodes, capable of measuring the oxygen consumption of single mouse and cattle embryos. Nevertheless, the prototype was not widely used because of limited sensitivity (Overström 1996). Furthermore, the measurements were time consuming and hence likely to interfere with the subsequent development of the embryo.

The oxygen consumption of small groups of mice (Houghton et al. 1996) and cattle embryos (Thompson et al. 1996) was also determined using an ultramicrofluorescence technique. This system employed the fluorescent characteristics of pyrene to indirectly measure the oxygen consumption of groups of embryos cultured in the presence of this substance over a period of $4-6 \mathrm{~h}$. This technique was unable to measure single embryos and, moreover, the exposure of the embryos to a fluorophore in the media may produce side-effects and would warrant further evaluations of possible toxic side-effects.

Trimachi et al. (2000a, 2000b) quantified the oxygen consumption of single zona-free mouse embryos with a self-referencing microelectrode technique, previously employed in the study of various biological systems (Land et al. 1999, Smith et al. 1999). In this technique, zona pellucidae were removed and thus the respiration rates reported might not accurately reflect the physiological oxygen consumption of intact embryos.

More recently, oxygen consumption of single bovine morulae and blastocysts was determined using scanning electrochemical microscopy (Shiku et al. 2001). The measurements required constant microscopic observation, and each embryo was held with holding pipettes in order to perform oxygen measurements at several defined positions because of the inhomogeneity observed at the blastocyst surface. However, the gradient generated by the oxygen consumption of the embryo was hyperbolic and consequently inherently difficult to measure. Furthermore, obtaining the overall respiration rate from several measurements on a single embryo is both inaccurate and time consuming.

An ideal method for measuring the oxygen respiration rate of single embryos should be non-invasive, rapid, simple, accurate, highly sensitive (capable of measuring individual embryos at any developmental stage), reproducible and not influence the subsequent development of the embryos.

In the present study, we introduce and have validated a novel high resolution microsensor system to determine oxygen consumption of single embryos with high sensitivity and accuracy. We have demonstrated that the system developed is suitable for non-invasive quantification of the differences in respiration rates of individual embryos at different developmental stages. We have further investigated whether individual respiration rates of in vitro produced day 7 blastocysts are correlated with morphological quality and sex or directly influenced by embryo diameter.

Preliminary results of this work have been presented earlier (Lopes et al. 2005).

\section{Materials and Methods}

All chemicals were purchased from Sigma (St Louis, MO, USA) unless otherwise stated.

\section{The nanorespirometer system}

The nanorespirometer system described below was purchased from Unisense A/S, Aarhus, Denmark (http://www. unisense.com). An overview of the complete system is given in Fig. 1. The oxygen measurement system consisted of an oxygen microsensor (OX50; tip diameter $40-60 \mu \mathrm{m})$, which is a miniaturized Clark-type oxygen sensor with a $90 \%$ response time of less than $5 \mathrm{~s}$ and an insignificant oxygen consumption. The sensor was mounted on a motorized micromanipulator (MM33), firmly attached to a stable laboratory stand (LS18) and connected to a high-sensitivity picoammeter (PA2000) capable of recording signals from the oxygen sensor with a resolution of $0.1 \mu \mathrm{mol} / \mathrm{l}$. Such sensor signals were, however, slightly sensitive to temperature changes $(2-3 \%$ signal increase per ${ }^{\circ} \mathrm{C}$ ). The picoammeter was connected to a computer running a data acquisition software (Profix version 2.2) via an $A / D$ converter (ADC-101). The software was used for reading and recording the data from the microsensor and for controlling the micromanipulator via a motor controller (MC-232).

The measuring unit (i.e. the rosette; Fig. 2b) consisted of seven fused glass capillaries (inner diameter $0.68 \mathrm{~mm}$, length $3 \mathrm{~mm}$ ) with a sealed glass bottom, attached to a UDEL polysulfone (Solvay Advanced Polymers L.L.C, Alpharetta, GA, USA) rosette disk. The rosette fits into a UDEL polysulfone rosette disk holder, which was attached 


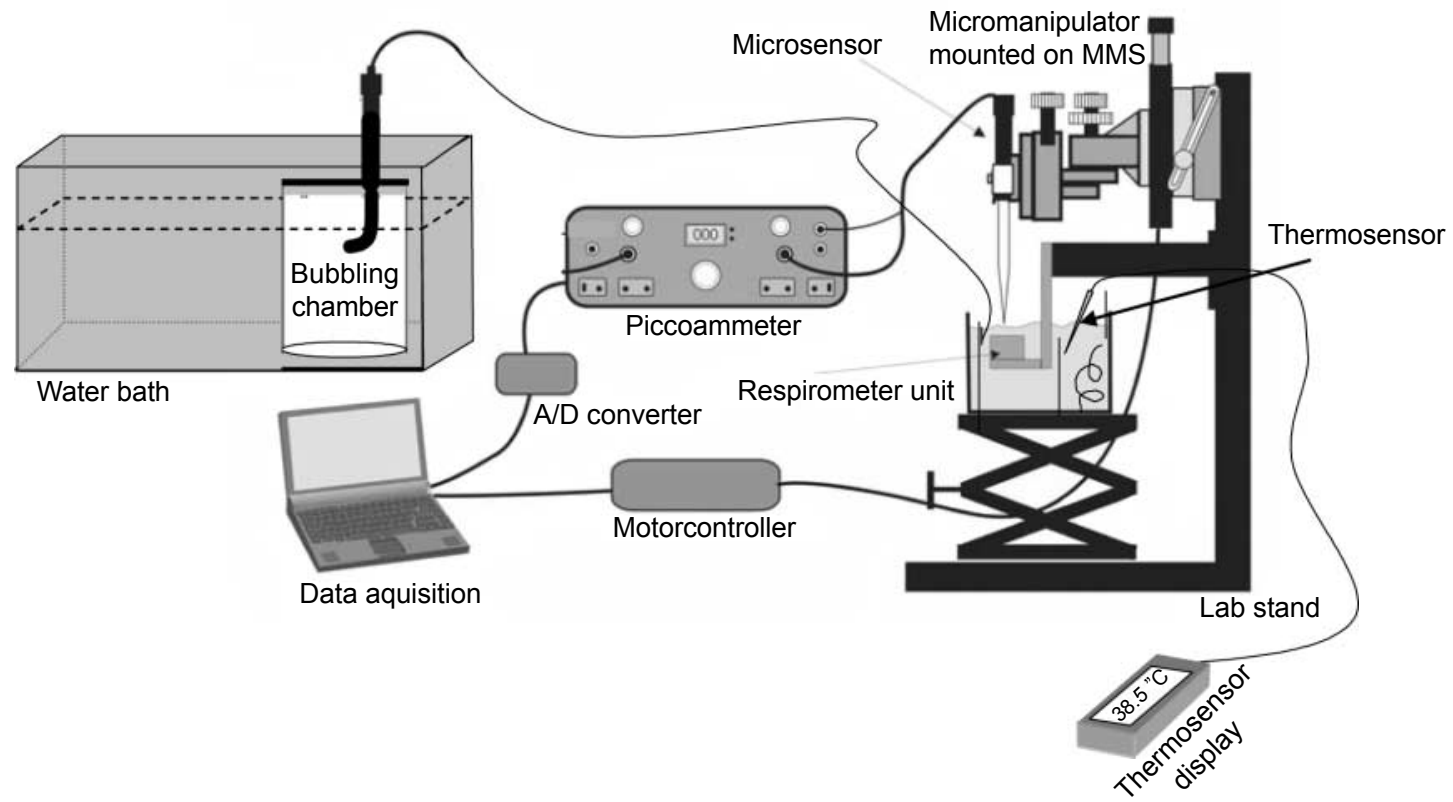

Figure 1 The complete nanorespirometer system. The temperature-controlled water bath is further connected by two silicone tubes (not shown in the picture) to the heating spiral in the glass incubation chamber. MMS; Micromanipulator system. to the laboratory stand by a metal frame. These three components formed the nanorespiration unit (Fig. 2C).

To ensure stable oxygen and temperature conditions during measurements, a large glass incubation chamber containing a heating spiral and a small beaker was fabricated for the purpose. The heating spiral was connected by two silicone tubes to a temperature-controlled water bath. The large glass incubation chamber filled with distilled water was placed on top of an elevator table and a magnetic stirrer. The temperature of the water in the incubator chamber was monitored by a thermosensor (TP 50; Unisense A/S) and maintained at $38.5^{\circ} \mathrm{C}$. Continuous circulation of hot water inside the glass incubation chamber was generated by bubbling with an air pump (AP50). The culture medium inside the beaker $(80 \mathrm{ml}$ SOFaaci; Holm et al. 1999) was composed of synthetic oviduct fluid med- ium with amino acids, citrate and inositol supplemented with antibiotics (gentamycin sulfate, $10 \mathrm{mg} / \mathrm{ml}$ ) and $5 \%$ cattle serum (CS; Danish Veterinary Institute, Frederiksberg, Denmark). This medium was held under a constant flow of humidified $5 \% \mathrm{CO}_{2}$ in $19 \% \mathrm{O}_{2}$ and in a semiclosed system, as the beaker was closed by a plastic cover with an opening in the centre. Gentle mixing of the medium inside the beaker was achieved with a small magnetic stirrer.

The oxygen microsensor was polarized for at least $2 \mathrm{~h}$ before a two-point calibration was performed. For the first point $\left(0 \% \mathrm{O}_{2}\right)$, ascorbic acid $(50 \mathrm{ml}$ sodium ascorbate in $50 \mathrm{ml} \mathrm{NaOH}, 0.1 \mathrm{M})$ was used. For the second point (19\% $\mathrm{O}_{2}$ ), calibration at the precise temperature, salinity and oxygen conditions experienced by the embryo during the
A

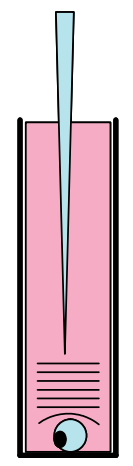

B

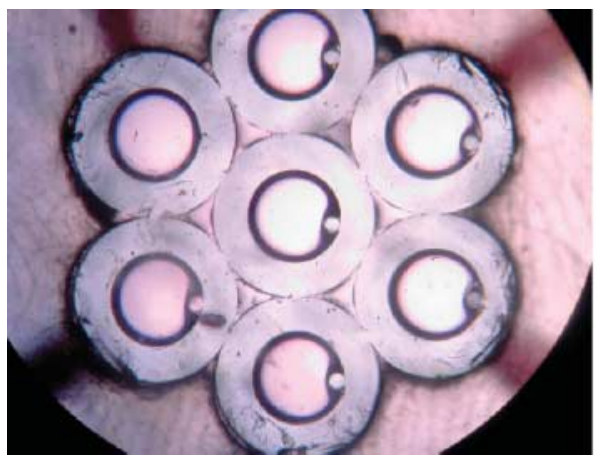

C

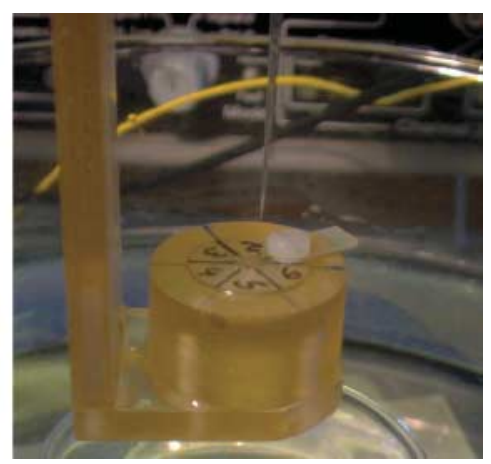

Figure 2 (A) Schematic drawing of a single rosette capillary with embryo, (B) seven fused glass capillaries comprising the 'rosette' seen from above and $(\mathrm{C})$ the nanorespiration unit. The rosette is mounted in the central rosette disc, which can be rotated to bring another capillary into alignment with the sensor. The numbers on the rosette disc are used to track which capillary is being investigated. The sensor is seen above the capillary. The polysulfone cylinder carrying the rosette disc and the rod to the left is the rosette disc holder, which is submerged into a beaker containing medium during measurement. 
actual measurements was performed by submerging the sensor in the medium inside the beaker.

Initially, the sensor was pre-positioned at the upper opening and centre (i.e. zero depth) of one of the glass capillaries in the rosette. This was performed manually using a dissection microscope (GZ4; Leica Microsystems, Wetzlar, Germany). Following this, the 'revolver' design of the system ensured that all other capillaries in the rosette were easily brought into position for profiling by rotating the rosette.

\section{In vitro embryo production}

The method used for in vitro production has been reported elsewhere (Holm et al. 1999). In brief, bovine immature cumulus-oocyte complexes (COCs) were aspirated from slaughterhouse-derived ovaries, selected (i.e. excluding denuded COCs and COCs with clear degenerative signs) and matured for $24 \mathrm{~h}$ in four-well dishes (Nunc, Roskilde, Denmark). Each well contained $400 \mu$ l bicarbonate-buffered TCM-199 medium (Gibco BRL, Paisley, Strathclyde, UK) supplemented with $15 \%$ CS, $10 \mathrm{IU} / \mathrm{ml}$ equine chorionic gonadotrophin and $5 \mathrm{IU} / \mathrm{ml}$ human chorionic gonadotrophin (Suigonan Vet; Intervet Scandinavia, Skovlunde, Denmark). The oocytes were matured under mineral oil at $38.5^{\circ} \mathrm{C}$ in $5 \% \mathrm{CO}_{2}$ in humidified air. Fertilization was performed in modified Tyrode's medium (Parrish et al. 1986) using frozen-thawed, Percoll-selected sperm. After $22 \mathrm{~h}$, cumulus cells were removed by vortexing and presumptive zygotes were transferred to $400 \mu \mathrm{l}$ culture medium and incubated at $38.5{ }^{\circ} \mathrm{C}$ in $5 \% \mathrm{CO}_{2}, 5 \% \mathrm{O}_{2}, 90 \% \mathrm{~N}_{2}$ atmosphere with maximum humidity. The embryos were in vitro produced over 12 independent replicates, by using approximately 50 oocytes per replicate.

\section{Embryo selection, evaluation and loading procedures}

On each day of experiment, five embryos at the eight-cell stage (day 3) or blastocyst stage (day 7) were selected randomly, to represent all morphological qualities, and individually transferred to one well of a four-well dish containing culture medium.

Day 3 embryos were selected based on the number of cells (preferably eight visible cells), uniformity of cell size, a healthy morphological appearance and absence of fragmentation.

Day 7 embryos were classified as either early, full, expanded, hatching or hatched blastocysts. Blastocysts were morphologically evaluated and given a quality score (quality 1, 2, 3 or 4) according to the definitions given by the International Embryo Transfer Society (IETS 1998). Furthermore, the diameter of each blastocyst was measured under a stereomicroscope (SMZ800; Nikon, Tokyo, Japan) using an ocular micrometer eyepiece.

Digital images of each embryo were acquired using a digital still camera (GC-X3E; JVC, Yokohama, Japan) mounted on an inverted optical microscope (TDM;
Nikon), with a thermal control microscope stage (CO 102; Linkam Scientific Instruments Ltd, Tadworth Surrey, UK).

Before loading the embryos, the sterile rosette was placed in a dish (Nunclon dishes; Nunc) containing $5 \mathrm{ml}$ pre-equilibrated culture medium. Each capillary of the rosette was manually filled with $1.1 \mu \mathrm{l}$ of the prepared incubation medium and the eventual air bubbles were removed.

Using a thin glass capillary pulled from a $2.5 \mathrm{ml}$ Pasteur pipette (BIE01141; Ar-Glas, Schott Glaswerke, Mainz, Germany), each of the selected embryos was collected from the four-well dish and loaded onto the bottom of each glass capillary in the rosette. One glass capillary in the rosette was left empty to serve as the reference measurement without respiratory activity.

The rosette was subsequently placed into the rosette disk holder with a pair of sterile tweezers. The elevator table was lifted until the rosette disk holder was submerged into the medium in the beaker. Finally, the semiclosed system was obtained by placing the plastic cover over the beaker. The system was left undisturbed until a steady-state linear oxygen gradient was established inside the glass capillaries of the rosette.

\section{Measurement of oxygen consumption}

The measurement principle employed in the nanorespirometer was based on measurement of linear steady-state oxygen gradients, generated by the respiration of the embryos in the glass capillaries of the rosette. The small internal dimensions of the glass capillaries and the viscosity of the medium established a diffusion-controlled system without turbulence, laminar flow or other bulk movements of the media. When the oxygen-consuming embryo was placed at the bottom of the capillary, the cylindrical shape of the diffusion space resulted in a largely linear concentration gradient when the system reached steady state. Using oxygen respiration values reported in the literature (Shiku et al. 2001) and the dimensions of the capillaries, it could be calculated that a typical embryo at the bottom of the capillary should reduce the oxygen concentration by about 25-50\% according to Ficks first law of diffusion (see section below). These predictions were subsequently verified by experimental measurement of the oxygen concentration near the embryo inside the glass capillary.

According to Crank (1997), a steady-state concentration gradient should be established in the capillary at time $t$, when $D t / I^{2}=0.45$, where $I$ is the capillary length and $D$ is the molecular diffusion coefficient for oxygen. Using the appropriate values for our measurement system we obtained an estimated time to generate a steady-state gradient of approximately 22 min, assuming that the surrounding system was in equilibrium and undisturbed by placing the embryo in the tube. To ensure a stable system before starting the measurements we decided to triple the required incubation time. 
After approximately $1 \mathrm{~h}$, the oxygen concentration gradient, generated by the respiration of the embryo (Fig. 2A), was determined by measuring the oxygen concentration at consecutive equidistant measurement points in the capillary. The recordings were made by moving the tip of the microsensor down through the media-filled glass capillary, measuring the signal in $200 \mu \mathrm{m}$ steps, up to a depth of $2500 \mu \mathrm{m}$ (Fig. 3). For each step, the sensor signal consisted of an average of 100 data points, recorded within $1 \mathrm{~s}$ after $2 \mathrm{~s}$ of equilibration. In order to acquire an oxygen concentration profile for the next embryo, the rosette disk was manually and gently rotated with the help of a sterile pipette to position the next capillary for profiling. In each round of measurements, all five embryos plus the empty capillary were measured. As a standard, two rounds of measurements were made, thus determining the respiration rate of each embryo twice.

\section{Calculation of the respiration rate based on the oxygen concentration profiles}

The basis for estimating respiration rates was the establishment of a significant decrease of oxygen partial pressure over the length of the tube, when placing a respiring embryo at the bottom. As the capillary dimensions are known, the linear oxygen gradient measured by the sensor can be used to estimate the oxygen flux towards the embryo, by applying Ficks first law of diffusion (Crank 1997):

$$
J=-D \frac{d c}{d x}
$$

where $d C / d x$ is the slope of the gradient, obtained from the recordings on the last ten steps (Fig. 3) and $D$ is the diffusion coefficient of the media. Under steady-state conditions, the oxygen flux will be equal to the respiration rate of the embryo in the capillary.

The solubility of oxygen in the medium (approximately $9 \%$ salinity and $37.0^{\circ} \mathrm{C}$ ) was calculated at $200 \mu \mathrm{mol} / \mathrm{l}$ (Garcia \& Gordon 1992). The diffusion coefficient in culture media at $38.5^{\circ} \mathrm{C}$ was likewise calculated to be

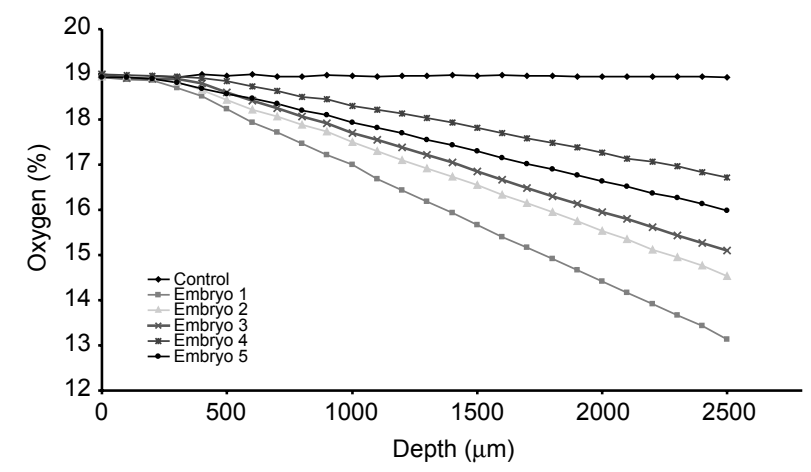

Figure 3 Recordings of oxygen gradients, generated by the respiration rate of five individual day 7 blastocyts. The control gradient corresponds to the measurement in the empty capillary and serves as negative reference ( $=$ no respiratory activity). The slope of each gradient is specific for each embryo.
$3.37 \times 10^{-5} \mathrm{~cm}^{2} / \mathrm{s} \quad(\mathrm{Li} \&$ Gregory 1974). Individual embryo respiration rates were expressed in $\mathrm{nl} \mathrm{O}_{2}$ /embryo per h (nl/h).

\section{Processing the embryos after the measurements and embryo lysis}

After the measurements, the rosette was removed from the rosette disk holder with a pair of tweezers and returned to the $40 \mathrm{~mm}$ dish with medium. The embryos were individually transferred from the rosette to a well of a four-well dish containing culture medium. Subsequently, individual embryos were transferred to a four-well dish containing $500 \mu \mathrm{l} \mathrm{Ca}^{2+}$-free and $\mathrm{Mg}^{2+}$-free phosphate-buffered saline (Biowhittaker, Walkersville, MD, USA) supplemented with $4 \mathrm{mg} / \mathrm{ml}$ polyvinylpyrrolidone, washed four times using a glass pipette and examined under a stereomicroscope to ensure that no cumulus cells were present. After this, each embryo was transferred under a stereomicroscope into a $0.5 \mathrm{ml}$ reaction tube containing dNTPs (Finnzymes, Espoo, Finland), $\mathrm{MgCl}_{2}$ (Finnzymes) and $0.1 \mathrm{mg} / \mathrm{ml}$ proteinase $\mathrm{K}$ (Boehringer Mannheim, Mannheim, Germany) in $10 \mu \mathrm{l}$ PCR buffer (DyNazyme buffer; Finnzymes). The mixture containing the embryos was overlaid with $25 \mu$ l DNAsefree mineral oil. The samples were subsequently incubated for $30 \mathrm{~min}$ at $37^{\circ} \mathrm{C}$ to release the DNA from the embryonic cells, and the proteinase $\mathrm{K}$ was then heat inactivated at $98^{\circ} \mathrm{C}$ for $8 \mathrm{~min}$. Finally, samples were stored at $4{ }^{\circ} \mathrm{C}$ until the sex diagnosis was performed.

\section{Embryo sexing by PCR}

The sex of the embryos was diagnosed by PCR (Saiki et al. 1988) on the basis of the presence or the absence of the Y-chromosomal ZFY gene and its X-chromosomal homologue, ZFX, as described earlier (Aasen \& Medrano 1990), but with modifications (Bredbacka \& Peippo 1992). Briefly, the lysed embryo samples were placed in the PCR machine and the temperature was increased to the initial denaturation step $\left(3 \mathrm{~min}\right.$ at $\left.94^{\circ} \mathrm{C}\right)$ during which the primers and the polymerase were added to each sample in $15 \mu \mathrm{l}$ PCR buffer. The final $25 \mu \mathrm{l}$ reaction mixture consisted of $10 \mathrm{mM}$ Tris $-\mathrm{HCl}\left(\mathrm{pH} 8.8\right.$ at $\left.25^{\circ} \mathrm{C}\right), 50 \mathrm{mM} \mathrm{KCl}$, $1.5 \mathrm{mM} \mathrm{MgCl}_{2}, 0.1 \%$ Triton X-100, $0.2 \mathrm{mM}$ dNTPs, $1.5 \mathrm{IU}$ DyNazyme DNA polymerase (Finnzymes) and $10 \mathrm{pmol}$ of both primers. After the initial denaturation step, the samples were amplified for 50 cycles as follows: $45 \mathrm{~s}$ at $94{ }^{\circ} \mathrm{C}, 35 \mathrm{~s}$ at $54{ }^{\circ} \mathrm{C}$ and $15-45 \mathrm{~s}$ at $72^{\circ} \mathrm{C}$. Finally, the samples were incubated for $5 \mathrm{~min}$ at $72^{\circ} \mathrm{C}$.

After amplification, $10 \mu \mathrm{l}$ of each PCR product was digested with $5 \mathrm{IU}$ Pstl (Roche Diagnostics GmbH, Mannheim, Germany) at $37^{\circ} \mathrm{C}$ for $1 \mathrm{~h}$. Digested PCR products were separated on $1.5 \%$ agarose gel (MetaPhor; FMC Bioproducts, Rockland, ME, USA) in $1 \times$ Tris-borate-EDTA buffer stained with $0.5 \mu \mathrm{g} / \mathrm{ml}$ ethidium bromide. The produced fragments were visualized under u.v. illumination. In cattle, Pstl cuts the ZFY homologue into two fragments (344 and $103 \mathrm{bp}$ ) whereas the PCR product of the ZFX 
homologue (445 bp) remains intact. The male samples were therefore recognized from the presence of three fragments (445, 344 and $103 \mathrm{bp}$ ) and the females from the presence of only one fragment (445 bp). As a quality control of the sex diagnosis, a non-template control and $10 \mathrm{pg}$ of female and male DNA were analyzed along with the embryo samples in each PCR run.

\section{Experimental design}

On each of the 12 days used for the experiment, one or two rosettes each containing five embryos and one empty glass capillary were measured twice. A total of 78 bovine embryos was measured at the eight-cell stage (day 3; $n=18$ ) and at various blastocyst stages (day $7 ; n=60$ ). Day 3 and day 7 embryos were measured on different experimental days. Subsequent sex diagnosis was performed on 64 of the 78 embryos (14 day 3 embryos; 50 day 7 embryos). Our standard in vitro production system (78\% and $42 \%$ as average cleavage and blastocyst rates respectively during the experimental period; Holm et al. 1999) served as a control for the quality of the embryos used.

\section{Statistical analysis}

Logistic regression (Reg Procedure; SAS Institute 1999) was used to determine the association between the first and second oxygen measurements of each embryo as well as the linearity of the oxygen gradient profiles.

Respiration rates were tested for normal distribution of the residual (Univariate Procedure; SAS Institute 1999). Based on these results, data were subsequently analyzed statistically using a mixed linear model (Mixed Procedure; SAS Institute 1999) including rosette, hours after fertilization, embryo diameter, stage, morphology, sex, as well as interaction of morphology and sex as systematic (fixed) factors. The random part of the model contained an interaction between rosette and date of measurement. Least square means (LSM) were produced by the previous model after removal of the interaction, as it was regarded as non-significant based on the results of the analysis.

The average respiration rate on days 3 and 7 was analyzed statistically using a mixed linear model (Mixed Procedure; SAS Institute 1999) including day as fixed effect. LSM were generated from the previous model. Data are expressed as LSM \pm S.E.

Factors affecting embryo diameter were assessed using a mixed linear model (Mixed Procedure; SAS Institute 1999). The model included rosette, hours after fertilization, embryo morphology and stage as fixed effects and interaction between rosette and date of measurement as random effects.

Sex ratios were compared with an expected 50:50 ratio by a corrected chi square procedure (Yate's adjustment; Box et al. 1978).

\section{Results}

\section{Sex ratio of embryos selected for oxygen} measurements

The proportion of male:female embryos was 64:36, which deviated significantly from the expected 50:50 ratio, both on day 3 and day 7 (Table $1 ; P<0.05$ ).

Among the 49 blastocysts sexed and morphologically evaluated (Table 2), the sex ratio previously observed (64:36) did not differ significantly between $(P>0.10)$ or within qualities $(P>0.05)$.

\section{Consistency and accuracy of the oxygen measurements}

The nanorespirometer system showed high accuracy, with an insignificant apparent oxygen consumption of $0.034 \pm 0.055 \mathrm{nl} / \mathrm{h} \quad(n=18)$ within the empty control capillaries. The gradients generated by the respiration of single embryos showed excellent linearity (Fig. 3), with a coefficient of determination $\left(r^{2}\right)$ of 0.999 . This linearity confirmed that an incubation period of $1 \mathrm{~h}$ was sufficient to establish stable conditions inside the glass capillaries of the rosette. Furthermore, measurements of each embryo were performed rapidly, with individual respiration rates being completed in $8 \mathrm{~min}$.

The results of the first and second oxygen measurements of the same embryos, performed $76 \pm 8 \mathrm{~min}$ apart, were in close agreement (Fig. 4), and the regression between the two data sets yielded a coefficient of determination $\left(r^{2}\right)$ of $0.9705(n=71 ; P<0.0001)$. Furthermore, the slope of the regression line was very close to one (i.e. 0.978 ), indicating a very slight drop of only $2.2 \%$ in respiration rate between the first and second measurements. Statistical analysis confirmed that the slope was not significantly different from one $(P>0.05)$ and the slight drop in rate from the first to the second measurement was thus an insignificant measurement inaccuracy.

The average respiration rate of the embryos was neither affected by the rosette used in the measurements (rosette 1 vs $2 ; P=0.69$ ) nor by the time after fertilization at which the measurements were performed $(P=0.31)$.

\section{Respiration rates of single embryos}

The average respiration rate was $0.38 \pm 0.011 \mathrm{nl} / \mathrm{h}$ for day 3 embryos $(n=18$; range $0.31-0.51 \mathrm{nl} / \mathrm{h})$, increasing

Table 1 Embryo stage and sex ratio of bovine in vitro produced embryos.

\begin{tabular}{lccl}
\hline Embryo stage & Day & No. of embryos & Male:female no. (ratio) \\
\hline Eight-cell & 3 & 14 & $9: 5 /\left(64: 36^{*}\right)$ \\
Blastocyst & 7 & 50 & $32: 18 /\left(64: 36^{*}\right)$ \\
\hline
\end{tabular}

* Significantly different from anticipated 50:50 ratio of males to females $(P<0.05)$. 
Table 2 Quality and sex ratio of day 7 bovine in vitro produced blastocysts*.

\begin{tabular}{lcc}
\hline Embryo quality & No. of embryos & Male:female no. (ratio) \\
\hline 1 & 13 & $11: 2 /(85: 15)$ \\
2 & 20 & $12: 8 /(60: 40)$ \\
3 & 12 & $8: 3 /(67: 33)$ \\
4 & 4 & $1: 3 /(25: 75)$ \\
\hline
\end{tabular}

* One collapsed blastocyst was not included in Table 2 due to difficulties in its classification.

significantly $(P<0.001)$ to $1.30 \pm 0.064 \mathrm{nl} / \mathrm{h}$ for day 7 embryos ( $n=60$; range $0.46-2.67 \mathrm{nl} / \mathrm{h}$ ) (Fig. 5).

\section{Effects of embryo morphology, sex, quality, diameter and developmental stage on respiration rates}

The diameter of the embryo was directly affected by embryonic stage $(P<0.01)$ and morphology $(P<0.01)$, as embryos of more advanced stages and of better quality were associated with a larger diameter.

Respiration rates were directly influenced by embryo diameter $(P<0.05)$, as larger embryos were associated with higher respiration rates $(y=9.06 \pm 3.71 \mathrm{nl} / \mathrm{h} \times \mathrm{mm}$; Fig. 6).

For day 7 blastocysts, respiration rates decreased proportionally to the morphological quality (Fig. 7), being $1.72 \pm 0.18^{\mathrm{a}}(n=18), 1.23 \pm 0.14^{\mathrm{b}}(n=23), 1.07 \pm 0.14^{\mathrm{b}}$ $(n=14)$ and $0.81 \pm 0.22^{\mathrm{b}}(n=4) \mathrm{nl} / \mathrm{h}$ for quality $1,2,3$ and 4 embryos respectively ( ${ }^{\mathrm{a}, \mathrm{b}}$ : $\left.P<0.01\right)$. Quality 1 embryos had the highest respiration rate, significantly different from all the other qualities, where respiration rate decreased less with each quality step (Fig. 7).

The average respiration rate of day 3 embryos was the same for males and females $(0.38 \pm 0.02 \mathrm{nl} / \mathrm{h})$. Similarly, in day 7 embryos the average respiration rate did not differ between sexes $(1.29 \pm 0.12 \mathrm{nl} / \mathrm{h}$ for females, $1.12 \pm 0.11 \mathrm{nl} / \mathrm{h}$ for males; $P=0.10)$.

\section{Discussion}

This study has provided experimental evidence that the new non-invasive microsensor technology described here was accurate, rapid and reproducible in measuring respir-

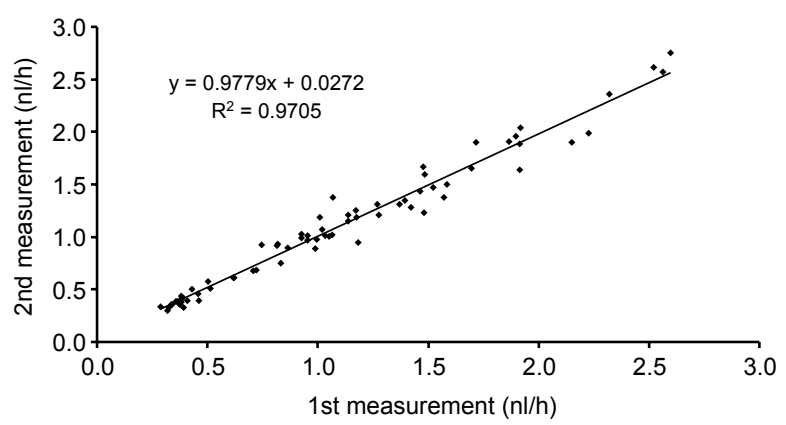

Figure 4 Relation between the first and second oxygen measurements, performed on the same embryos with $76 \pm 8$ min interval.

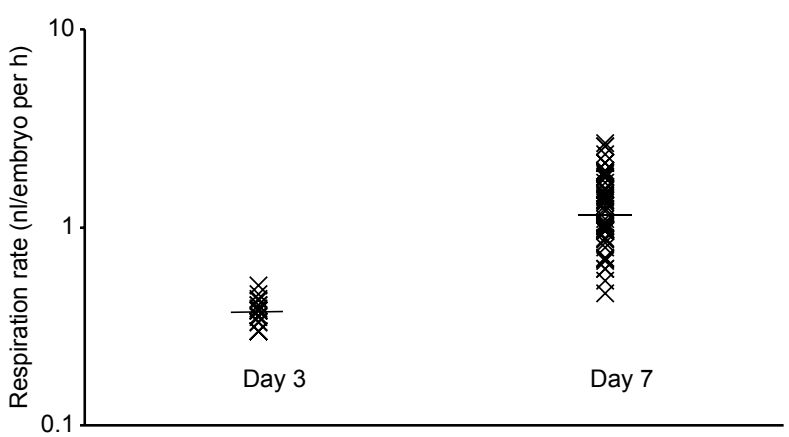

Figure 5 Respiration rates for single day $3(n=18)$ and day 7 $(n=60)$ embryos. The lines in the figure represent the average respiration rate for each embryo stage.

ation rates of individual bovine embryos. The excellent linearity exhibited by the oxygen gradients and the close relation between first and second measurements demonstrated the consistency of the results obtained. In fact, the concentration gradient may deviate from perfect linearity in close proximity to the embryo or to the open top of the capillary. However, as our measurements were performed more than $500 \mu \mathrm{m}$ from the embryo and from the top, these deviations were negligible and did not affect the linearity of the gradients. Moreover, the small dimensions of the sensor used did not disrupt the oxygen flux towards the embryo and did not interfere with the oxygen gradients generated by embryo respiration, as is apparent from the linear oxygen gradients measured. A constant flow of humidified $5 \% \mathrm{CO}_{2}$ in $19 \% \mathrm{O}_{2}$ was employed in order to ensure stable oxygen conditions, since the measurements were performed under a semi-closed system and 19\% $\mathrm{O}_{2}$ is closer to normal atmospheric conditions $\left(21 \% \mathrm{O}_{2}\right)$. Even though previous studies have reported that oxygen concentrations used during culture can influence embryo development, cell numbers and gene expression (Harvey et al. 2004), we believe that the transfer for a relative short period to a different $\mathrm{O}_{2}$ concentration medium during the oxygen measurements did not have a significant impact on the metabolism of the embryos. A slight evaporation of the medium inside the beaker was noticed

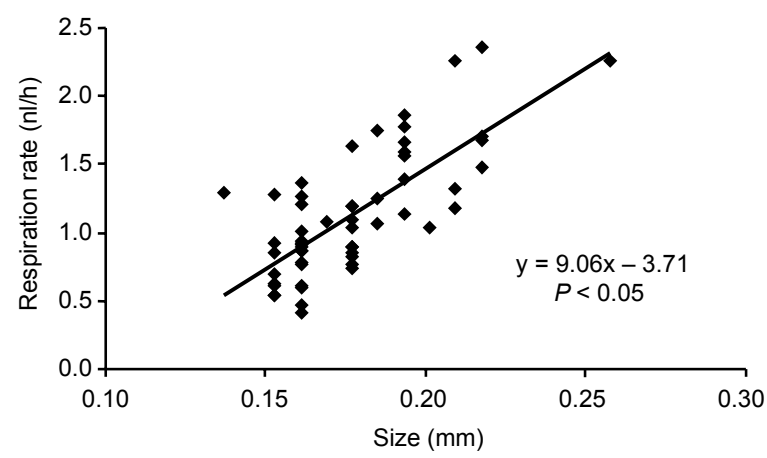

Figure 6 Relation between respiration rates and diameter of day 7 in vitro-produced bovine blastocysts. 


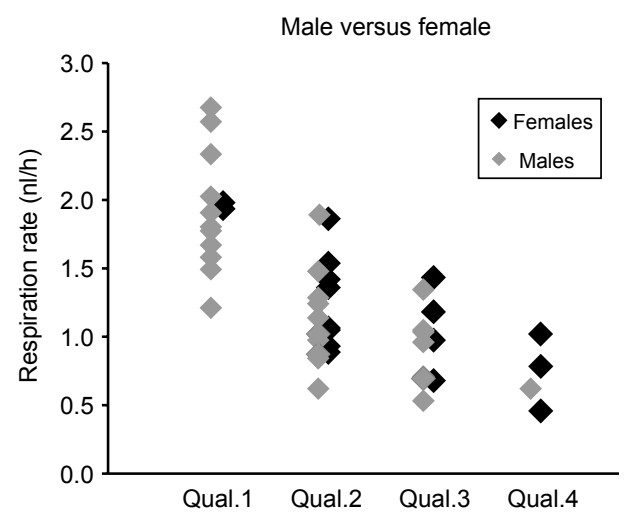

Figure 7 Respiration rates of day 7 in vitro-produced bovine blastocysts, according to their quality (Qual.) and sex.

throughout the measurements. Nevertheless, the rosettes were totally submerged in a large volume of media $(80 \mathrm{ml})$ and as the surface-to-volume ratio of such a large volume is relatively low, there is only insignificant evaporation of the large media volume.

The high resolution of the system together with the insignificant effect of rosette changes are further advantages of this technology. The negligible yet persistent oxygen consumption observed in the control capillaries could possibly be explained by the slight oxygen consumption of the sensor during the time required to conduct the measurement, as the electrode current of about $100 \mathrm{pA}$ corresponds to an oxygen consumption of about $0.02 \mathrm{nl} / \mathrm{h}$. However, as the sensor was only transiently inserted in the capillaries and did not have time to alter the concentration significantly, the effect was more likely due to slight fluctuations in temperature (i.e. less than $0.5^{\circ} \mathrm{C}$ ) observed throughout the measurements. In this study, the time necessary for measuring each embryo was approximately $8 \mathrm{~min}$, although currently it is possible to perform single measurements in 2 min due to further development of the technology.

The microsensor technology described here measures the overall respiration rate of a single embryo, based on a gradient generated by the whole embryo lying freely on the bottom of a glass capillary. This overcomes the problem of the inhomogeneity of the blastocyst surface, encountered by Shiku et al. (2001) when using the scanning electrochemical microscopy (SCEM) technique. In that paper, the authors reported that the oxygen consumption rate of a blastocyst varied, with higher oxygen consumption rates being observed in the inner cell mass side when compared with the trophoblast side. A distinct advantage of our technology was the linear concentration gradient within the capillary so the measurement does not require exact knowledge (i.e. with $\mu \mathrm{m}$ accuracy) of the radial distance from the surface of the embryo to the sensor tip, and measurements do not need to be made in very close proximity to the embryo. On the contrary, with the SCEM technique spherical diffusion establishes a hyper- bolic concentration difference around the embryo and the measurements thus had to be carried out in exactly the same location and close to the embryo surface for all embryos, in order to establish a direct comparison between oxygen consumption rates of different embryos.

The respiration rates observed here for day 3 embryos were slightly higher than those reported by Thompson et al. (1996) for embryos of similar age. The average respiration rate of day 7 blastocysts, measured by the microsensor system, was in accordance with the results obtained by Overström (1996), using in vivo day 7 bovine blastocysts $(1.47 \pm 0.44 \mathrm{nl} / \mathrm{h})$. On the other hand, our results for day 7 embryos were considerably lower than those documented by Donnay \& Leese (1999), Kaidi et al. (2001) and Shiku et al. (2001), but their studies were performed on day 8 blastocysts, containing higher number of cells and larger diameters. Differences between our data and those of Thompson et al. (1996) can also be explained by the different blastocyst stage (day 7 versus day 6) used in these two experiments. According to Eckert et al. (1998), exogenously added protein significantly alters the oxidative metabolism of the embryos. Thus, it is likely that the type of medium used in our experiment had an influence on the results.

In the present study, respiration rates of day 7 blastocysts were approximately 3.4-fold higher than those observed for eight-cell stage embryos (Fig. 5). These values are in agreement with the 2.8-fold increase between morula and blastocyst reported by Houghton et al. (1996) for murine embryos. Similarly, Thompson et al. (1996) showed a 3.3-fold increase in oxygen consumption by day 6 bovine blastocysts over 16-cell morula, and Trimarchi et al. (2000a) confirmed that blastocyts consume two to four times more oxygen than do cleavage stage embryos. Indeed, such an increase has already been observed in the early Cartesian diver techniques (Fridhandler et al. 1957, Mills \& Brinster 1967), where groups of blastocysts consumed markedly more oxygen than groups of cleavage stage embryos.

The increase in respiration rate as the preimplantation embryo develops to more advanced stages has been associated with the increase in energy demand for the initiation of the processes of compaction and blastocyst formation (Thompson et al. 1996, Trimarchi et al. 2000a, Harvey et al. 2002, 2004). This increase of metabolic activity was also supported in our study, by correlating the low respiration rates of eight-cell embryos with their low ATP demand, and the higher respiration rates of day 7 blastocysts with an increase in ATP demand, due to the presence of an active embryonic genome as well as to changes in mitochondria morphology and number (Trimarchi et al. 2000a). Part of the ATP produced at the blastocyst level is the result of increased glycolysis and is therefore not related to oxygen consumption; nevertheless $85 \%$ of the total ATP produced by the blastocyst is still generated by mitochondrial oxidative phosphorylation, a process requiring oxygen (Benos \& Baladan 1983, Leese 
2003). According to Trimarchi et al. (2000a), the oxidative phosphorylation-independent oxygen consumption remains constant throughout embryo development (Trimarchi et al. 2000a). Therefore, it is likely that the observed increase in oxygen consumption mostly reflects an increase in oxidative phosphorylation at the blastocyst stage.

Respiration rates of day 7 in vitro blastocysts decreased with morphological quality and embryo diameter. Nevertheless, in terms of morphology, only quality 1 embryos were significantly different from all the other qualities, showing the highest respiration rates. The significance of quality 1 embryos having higher respiration rates over that of the other classes was confirmed with a power analysis, which also showed that differences between the other quality classes could not be detected even in larger experiments (A S Lopes and P Løvendahl, unpublished observations).

Our results were consistent with those reported by Magnusson et al. (1986), showing decreasing respiration rates for healthy looking, pathologically cleaved and degenerated human blastocysts. Furthermore, this study is supported by the work of Shiku et al. (2001) using bovine embryos, but is in contrast to the results of Overström (1992) who observed no correlation between in vivo bovine embryo morphology and respiration. These differences might be related to the different measuring systems employed and with the source and stage of the embryos used.

Unfortunately only a relatively small number of quality 4 blastocysts were investigated so that statistically significant associations between embryo respiration and certain morphological qualities could not be adequately demonstrated. Similarly, no significant differences were detected between the two intermediate morphological grades (quality 2 and 3). This was expected since morphological evaluation of these embryo types is particularly difficult and embryos are often unintentionally under/over graded. Furthermore, we have observed considerable variation in respiration rates within morphologies, as previously described in other studies (Overström 1992, Shiku et al. 2001). Together, these results partly sustain the hypothesis that embryos with a higher level of metabolism are most often of higher morphological quality, but the observed variation between respiration rates and morphological quality indicates that respiration rate measurements may be a quality indicator on its own.

The present data revealed that blastocysts in more advanced stages of development had a significantly larger diameter and, consequently, higher respiration rates. This observation may be explained by the fact that the activity of the $\mathrm{Na}^{+}-\mathrm{K}^{+}$-ATPase accounts for most of the energy consumption of the blastocyst (Donnay \& Leese 1999), so it is expected that the largest blastocysts will consume more oxygen. Direct correlations between embryo diameter and respiration rate have not been described in other studies. On the other hand, correlations between blasto- cyst stage and the corresponding oxygen consumption have been reported for in vivo bovine (Thompson et al. 1995) and in vitro porcine embryos (Sturmey \& Leese 2003). In these studies, measurements of both early and expanded blastocysts confirmed that oxygen consumption decreased after the blastocyst had completed expansion. In contrast, Donnay \& Leese (1999) demonstrated that the oxygen uptake was directly correlated with the degree of re-expansion of the bovine blastocysts and that hatching blastocysts showed the highest oxygen consumption. Thus, whether or not more advanced bovine blastocyst stages exhibit considerable lower oxygen consumption requires further investigation.

We were not able to adequately resolve possible differences in respiration rates depending on the sex of the embryo. The respiration rates were similar for male and female blastocysts, regardless of the uneven male:female distribution within quality categories. To our knowledge, there are no data available addressing the issue of respiration rate among embryos of different sexes. As Fig. 6 suggests, it is possible that once a female embryo reaches a stage and morphology that would enable us to characterize it as belonging to a certain quality, its respiration rate would be equal to that of a male embryo.

A sex ratio of $64 \%$ males was observed among the embryos selected for the oxygen measurements. This skewed sexed ratio was not observed in a previous work, using the same culture conditions (Holm et al. 1998). However, the selection of embryos for sexing was performed differently in these studies. Our results might therefore be in accordance with other studies, indicating that glucose in the medium possibly favours a more rapid cleavage and growth of male embryos over females (Bredbacka \& Bredbacka 1996, Peippo et al. 2001), the latter having a slower cleaving process (Avery et al. 1991, 1992) and being less capable of progressing from the morula/early blastocyst stage to more advanced stages of development (Larson et al. 2001). Nevertheless, the study of sex ratio differences was beyond the scope of this experiment.

The novel high resolution microsensor technology evaluated in this study enables a non-invasive, simple, accurate and consistent measurement of the respiration rate of a single embryo in a period of $8 \mathrm{~min}$ or less. These characteristics make this technology promising for assessing embryo quality in the future. However, whether respiration rates of individual embryos, as measured by the microsensor technology presented here, will prove to be an accurate indicator of subsequent embryo viability, requires further investigations, involving embryo transfer (authors' work in progress).

We support the general idea (Overström 1992, 1996, Boiso et al. 2002, Abe \& Hoshi 2003, Neuber et al. 2003, Scott 2003) that a more objective measurement of embryo quality is required and is undoubtedly a key to overcome the shortcomings of morphological evaluations, such as subjectivity. The objectivity of the microsensor technology, 
combined with the traditional and accepted morphological evaluation, might prove to be a very accurate method for improving embryo classification and subsequent selection. The improved selection, based on a combination of these two evaluation methods, might have the potential to help reduce financial losses in animal embryo transfer units, as well as avoiding a large number of unsuccessful pregnancies and multiple births in human in vitro fertilization clinics.

\section{Acknowledgements}

The authors wish to thank Anette Pedersen, Elin La Coste, Klaus Villemoes, Lars Borregard Pedersen and Ruth Kristensen for their technical assistance. Novo Nordisk Foundation financially supported the acquisition of the microsensor technology and the Danish Directorate for Food, Fisheries and Agri Business (TFH03-8) the embryo work. A S Lopes is supported by a grant from Foundation for Science and Technology (FCT), Ministry of Science and Technology, Portugal (SFRH/BD/8486/2002). The authors declare that there is no conflict of interest that would prejudice the impartiality of this scientific work.

\section{References}

Aasen E \& Medrano JF 1990 Amplification of the ZFY and ZFX genes for sex identification in humans, cattle, sheep and goats. Biotechnology 8 1279-1281.

Abe H \& Hoshi H 2003 Evaluation of bovine embryos produced in high performance serum-free media. Journal of Reproductive Development 49 193-202.

Avery B, Madison V \& Greve T 1991 Sex and development in bovine in-vitro fertilized embryos. Theriogenology 35 953-963.

Avery B, Jorgensen CB, Madison V \& Greve T 1992 Morphological development and sex of bovine in vitro-fertilized embryos. Molecular Reproduction and Development 32 265-270.

Benos DJ \& Baladan RS 1983 Energy metabolism of preimplantation mammalian blastocysts. American Journal of Physiology 245 40-45.

Boiso I, Veiga A \& Edwards RG 2002 Fundamentals of human embryonic growth in vitro and the selection of high-quality embryos for transfer. Reproductive Biomedicine OnLine $\mathbf{5}$ 328-350.

Box G, Hunter WG \& Hunter JS 1978 In Statistics for Experimenters, edn 1, pp 150. New York: John Wiley \& Sons.

Bredbacka P \& Peippo J 1992 Sex diagnosis of ovine and bovine embryos by enzymatic amplification and digestion of DNA from the ZFY/ZFX locus. Agricultural Science of Finland $1233-238$.

Bredbacka K \& Bredbacka P 1996 Sex-related cleavage rate difference in bovine embryos produced in vitro is controlled by glucose. Theriogenology 45191 (abstract).

Crank J 1997 In The Mathematics of Diffusion, edn 2, pp 2-51. Oxford: Oxford Science Publications.

Donnay I \& Leese HJ 1999 Embryo metabolism during the expansion of the bovine blastocyst. Molecular Reproduction and Development 53 171-178.

Eckert J, Pugh PA, Thompson JG, Niemann H \& Tervit HR 1998 Exogenous protein affects development competence and metabolic activity of bovine preimplantation embryos in vitro. Reproduction, Fertility and Development 10 327-332.

Farin PW, Britt JH, Shaw DW \& Slenning BD 1995 Agreement among evaluators of bovine embryos produced in vivo or in vitro. Theriogenology 44 339-349.
Fridhandler I, Hafez ESE \& Pincus G 1957 Developmental changes in the respiratory activity of rabbit ova. Experiments in Cell Research 13 132-139.

Garcia H \& Gordon L 1992 Oxygen solubility in seawater: better fitting equations. Limnology and Oceanography 37 1307-1312.

Gardner DK 1999 Development of serum-free culture systems for the ruminant embryo and subsequent assessment of embryo viability. Journal of Reproduction and Fertility 54 (Suppl) 461-475.

Gopichandran N \& Leese HJ 2003 Metabolic characterization of the bovine blastocyst, inner cell mass, trophectoderm and blastocoel fluid. Reproduction 126 299-308.

Harvey AJ, Kind KL \& Thompson JG 2002 REDOX regulation of early embryo development. Reproduction 123 479-486.

Harvey AJ, Kind KL, Pantaleon M, Armstrong DT \& Thompson JG 2004 Oxygen-regulated gene expression in bovine blastocysts. Biology of Reproduction 71 1108-1119.

Hasler JF 1998 The current status of oocyte recovery, in vitro embryo production, and embryo transfer in domestic animals, with an emphasis on the bovine. Journal of Animal Science 76 (Suppl 3) 52-74.

Holm P, Shukri NN, Vajta G, Booth P, Bendixen C \& Callesen H 1998 Developmental kinetics of the first cell cycles of bovine in vitro produced embryos in relation to their in vitro viability and sex. Theriogenology $\mathbf{5 0} 1285-1299$.

Holm P, Booth PJ, Schmidt MH, Greve T \& Callesen H 1999 High bovine blastocyst development in a static in vitro production system using SOFaa medium supplemented with sodium citrate and myo-inositol with or without serum-proteins. Theriogenology 52 $683-700$

Houghton FD, Thompson JG, Kennedy CJ \& Leese HJ 1996 Oxygen consumption and energy metabolism of the early mouse embryo. Molecular Reproduction and Development $\mathbf{4 4}$ 476-485.

Houghton FD, Humpherson PG, Hawkhead JA, Hall CJ \& Leese HJ $2003 \mathrm{Na}+, \mathrm{K}+$, ATPase activity in the human and bovine preimplantation embryo. Developmental Biology 263 360-366.

IETS 1998 In Manual of the International Embryo Transfer Society, edn 3, pp 167-170. Eds DA Stringfellow \& SM Seidel. Savoy, IL, USA: International Embryo Transfer Society.

Kaidi S, Bernard S, Lambert P, Massip A, Dessy F \& Donnay I 2001 Effect of conventional controlled-rate freezing and vitrification on morphology and metabolism of bovine blastocysts produced in vitro. Biology of Reproduction 65 1127-1134.

Land SC, Porterfield DM, Sanger RH \& Smith PJ 1999 The self-referencing oxygen-selective microelectrode: detection of transmembrane oxygen flux from single cells. Journal of Experimental Biology 202 211-218.

Larson MA, Kimura K, Kubisch HM \& Roberts RM 2001 Sexual dimorphism among bovine embryos in their ability to make the transition to expanded blastocyst and in the expression of the signaling molecule IFN-tau. PNAS 98 9677-9682.

Leese HJ 1991 Metabolism of the preimplantation mammalian embryo. Oxford Reviews in Reproductive Biology 13 35-72.

Leese H 2003 What does an embryo need? Human Fertility 6 $180-185$

Leese HJ \& Barton AM 1984 Pyruvate and glucose uptake by mouse ova and preimplantation embryos. Reproduction and Fertility $\mathbf{7 2}$ 9-13.

Li YH \& Gregory S 1974 Diffusion of ions in seawater and deep sea sediments. Geochimica et Cosmochimica Acta 38 703-714.

Lopes AS, Ramsing N, Larsen LH, Räty M, Peippo J, Greve T \& Callesen H 2005 Correlation between oxygen respiration rates and morphology, sex, diameter and developmental stage of single bovine IVP-embryos. Reproduction, Fertility and Development 17 151 (abstract).

Magnusson C, Hillensjo T, Hamberger L \& Nilsson L 1986 Oxygen consumption by human oocytes and blastocysts grown in vitro. Human Reproduction 1 183-184.

Mills RM \& Brinster RL 1967 Oxygen consumption of preimplantation mouse embryos. Experimental Cell Research 47 337-344. 
Neuber E, Rinaudo P, Trimarchi JR \& Sakkas D 2003 Sequential assessment of individually cultured human embryos as an indicator of subsequent good quality blastocyst development. Human Reproduction 18 1307-1312.

Nilsson BO, Magnusson C, Widehn S \& Hillensjo T 1982 Correlation between blastocyst oxygen consumption and trophoblast cytochrome oxidase reaction at initiation of implantation of delayed mouse blastocysts. Journal of Embryology and Experimental Morphology 71 75-82.

Overström EW 1987 In vitro assessment of blastocyst differentiation. In The Mammalian Preimplantation Embryo, edn 1, pp 95-116. Ed. BD Bavister. New York: Plenum Press.

Overström EW 1992 Manipulation of early embryonic development. Animal Reproduction Science 28 277-285.

Overström EW 1996 In vitro assessment of embryo viability. Theriogenology 45 3-16.

Overström EW, Duby RT, Dobrinsky J, Roche JF \& Boland MP 1992 Viability and oxidative metabolism of the bovine blastocyst. Theriogenology 37269 (abstract).

Parrish JJ, Susko-Parrish JL, Liebfried-Rutledge ML, Critser ES, Eyestn WH \& First NL 1986 Bovine in vitro fertilization with frozenthawed semen. Theriogenology 25 51-600.

Peippo J, Kurkilahti M \& Bredbacka P 2001 Developmental kinetics of in vitro produced bovine embryos: the effect of sex, glucose and exposure to time-lapse environment. Zygote 9 105-113.

Rieger D 1992 Relationships between energy metabolism and development of early mammalian embryos. Theriogenology 37 75-93.

Rieger D \& Loskutoff NM 1994 Changes in the metabolism of glucose, pyruvate, glutamine and glycine during maturation of cattle oocytes in vitro. Journal of Reproduction and Fertility $\mathbf{1 0 0}$ $257-262$.

Rieger D, Loskutoff NM \& Betteridge KJ 1992 Developmentally related changes in the metabolism of glucose and glutamine by cattle embryos produced and co-cultured in vitro. Journal of Reproduction and Fertility 95 585-595.

Saiki RK, Gelfand DH, Stoffel S, Scharf SJ, Higuchi R, Horn GT, Mullis KB \& Erlich HA 1988 Primer-directed enzymatic amplification of DNA with a thermostable DNA polymerase. Science $\mathbf{2 3 9}$ 487-491.
SAS Institute 1999 In SAS/STAT User's Guide (Release 8.2). Cary, NC: Statistical Analysis System Institute Inc.

Scott L 2003 The biological basis of non-invasive strategies for selection of human oocytes and embryos. Human Reproduction Update $9237-249$.

Shiku H, Shiraishi T, Ohya H, Matsue T, Abe H, Hoshi H \& Kobayashi M 2001 Oxygen consumption of single bovine embryos probed by scanning electrochemical microscopy. Analytical Chemistry $\mathbf{7 3}$ $3751-3758$.

Smith PJ, Hammar K, Porterfield DM, Sanger RH \& Trimarchi JR 1999 Self-referencing, non-invasive, ion selective electrode for single cell detection of trans-plasma membrane calcium flux. Microscopic Research Techniques 46 398-417.

Sturmey RG \& Leese HJ 2003 Energy metabolism in pig oocytes and early embryos. Reproduction 126 197-204.

Thompson JG 2000 In vitro culture and embryo metabolism of cattle and sheep embryos - a decade of achievement. Animal Reproduction Science 60-61 263-275.

Thompson JG, Partridge RJ, Houghton FD, Kennedy CJ, Pullar D, Wrathall AE \& Leese HJ 1995 Preliminary observations of the uptake of oxygen by day 7 bovine blastocysts. Theriogenology 43 337 (abstract).

Thompson JG, Partridge RJ, Houghton FD, Cox CI \& Leese HJ 1996 Oxygen uptake and carbohydrate metabolism by in vitro derived bovine embryos. Journal of Reproduction and Fertility 106 299-306.

Trimarchi JR, Liu L, Porterfield DM, Smith PJ \& Keefe DL 2000a Oxidative phosphorylation-dependent and -independent oxygen consumption by individual preimplantation mouse embryos. Biology of Reproduction 62 1866-1874.

Trimarchi JR, Liu L, Porterfield DM, Smith PJ \& Keefe DL $2000 \mathrm{~b}$ A non-invasive method for measuring preimplantation embryo physiology. Zygote 8 15-24.

Received 23 February 2005

First decision 10 May 2005

Revised manuscript received 11 June 2005

Accepted 29 June 2005 